\title{
A Novel Small-Caliber Bacterial Cellulose Vascular Prosthesis: Production, Characterization, and Preliminary In Vivo Testing
}

\author{
Alexandre F. Leitão, Miguel A. Faria, Augusto M. R. Faustino, \\ Ricardo Moreira, Petra Mela, Luís Loureiro, Ivone Silva, Miguel Gama*
}

Vascular grafts are used to bypass damaged or diseased blood vessels. Bacterial cellulose (BC) has been studied for use as an off-the-shelf graft. Herein, we present a novel, cost-effective, method for the production of small caliber BC grafts with minimal processing or requirements. The morphology of the graft wall produced a tensile strength above that of native vessels, performing similarly to the current commercial alternatives. As a result of the production method, the luminal surface of the graft presents similar topography to that of native vessels. We have also studied the in vivo behavior of these BC graft in order to further demonstrate their viability. In these preliminary studies, 1 month patency was achieved, with the presence of neovessels and endothelial cells on the luminal surface of

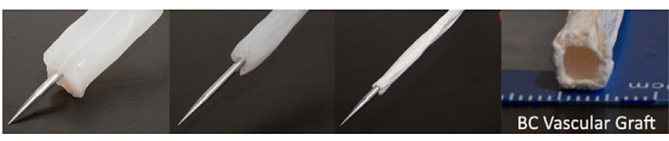
the graft.

M. Gama, A. F. Leitão

CEB-Centre of Biological Engineering, University of Minho, Braga 4710-057, Portugal

E-mail: fmgama@deb.uminho.pt

M. A. Faria

Departamento de Clínicas Veterinárias; Laboratório de Farmacologia e Neurobiologia; Unidade Multidisciplinar de Investigação Biomédica (UMIB), ICBAS- Biomedical Sciences Institute Abel Salazar, University of Porto, Oporto 4050-313, Portugal

A. M. R. Faustino

ICBAS- Biomedical Sciences Institute Abel Salazar, University of Porto, Oporto 4050-313, Portugal

R. Moreira, P. Mela

Department of Tissue Engineering \& Textile Implants, AME-

Helmholtz Institute for Biomedical Engineering, D-52074 Aachen, Germany

L. Loureiro, I. Silva

Serviço de Angiologia e Cirurgia Vascular, Centro Hospitalar do

Porto, Oporto 4050-313, Portugal

\section{Introduction}

Data from the World Health Organization (WHO) suggest that the number of people that suffer from cardiovascular diseases (CVDs) will increase from 17.3, in 2008, to 23.3 million by 2030 . In vascular surgery, surgical bypass is fundamental in the treatment of some diseases. Vascular grafts replace, bypass or maintain function of damaged, occluded or diseased blood vessels. The chosen conduit, and its success, depends on several factors such as availability, size, ease of handling and technical facility, thrombogenicity, resistance to infection and dilation, durability, longterm patency, and price. ${ }^{[1,2]}$

The most common, and generally considered ideal grafts, are autografts, i.e., vessels collected from the patient. There are generally two major downsides to the use of autografts. The first is the need for additional surgical interventions, before the actual bypass surgeries, in order to collect the autologous vessel that will then be used as the bypass graft. 
Along with this, the actual availability of these vessels may be limited, due to prior interventions. Secondly, these grafts are subject to the natural progression of the patient's disease which can lead to deterioration, over time, of the biological grafts ${ }^{[3]}$ and so synthetic grafts have been used as cardiovascular grafts.

Polyethylene terephthalate (PET or Dacron) and expanded polytetrafluoroethylene (ePTFE) grafts are the gold standard, at the moment, for synthetic grafts in large and medium diameter $(>6 \mathrm{~mm})$ vessels. However, these synthetic alternatives are not yet the ideal solution. While demonstrating acceptable results in larger diameter grafts, as internal diameter decreases so does its long-term patency. ${ }^{[4,5]}$ These grafts ultimately fail under $6 \mathrm{~mm}$ in diameter, due to neointimal hyperplasia and/or thrombosis. ${ }^{[6,7]}$

A large amount of work has, therefore, been aimed at finding alternative solutions to these materials through either natural or synthetic polymer materials. ${ }^{[2,8-10]}$ The field is ever growing and the lack of a clear optimal polymer has led, and continues to lead, to many potential options, either by further developing already existing grafts (PET and ePTFE) or creating new non-degradable grafts of polyurethane, polyvinyl alcohol, or bacterial cellulose (BC). Another option is researching and developing temporary grafts that ultimately allow for a tissue engineered solution, either by prior cell-seeding or allowing for native cell migration, attachment, and ingrowth. Ultimately, these tissue engineered grafts would end up producing a new vessel out of native tissue from a scaffold made of, e.g., collagen, fibrin, elastin, silk, and polycaprolactone. ${ }^{[11-14]}$

One promising candidate is $\mathrm{BC}$. This polymer is a highly pure linear polysaccharide, consisting of $\beta(1 \rightarrow 4)$-linked d-glucose monomers. It is secreted by bacteria of the Gluconacetobacter genus. Once secreted, it forms a fibrous network hydrogel that has been extensively studied for biomedical applications due to its morphology, high purity, water-holding capacity, tensile strength, malleability, and biocompatibility ${ }^{[15-17]}$ and has been proposed for applications such as wound dressings, ${ }^{[18]}$ artificial skin ${ }^{[19]}$ or blood vessels, ${ }^{[20-22]}$ and as a scaffold for tissue engineering. ${ }^{[17,23,24]}$ The hemocompatibility of BC has been comprehensively studied in our group, with rather promising results. ${ }^{[19]}$

Many methods have been proposed and patented ${ }^{[25-32]}$ for the production of $\mathrm{BC}$ tubular grafts. Generally, the methods employed take advantage of the way the $\mathrm{BC}$ hydrogel is produced at air-medium interfaces. ${ }^{[16,33]} \mathrm{How}^{-}$ ever, these production methods require dedicated bioreactors specifically designed for graft production. An oxygen permeable support scaffold provides a three-dimensional surface upon which BC is produced. The thickness and density of the BC structures produced by this method is limited, due to the oxygen rich environment produced being limited to the immediate vicinity of that oxygen permeable silicone tube. Additionally, oxygen must permeate consistently and homogenously throughout the silicone otherwise the thickness of the produced BC will not be homogenous. Also, some cases develop interconnected sheets or layers of $B C$ in the wall of the graft. These sheets can potentially delaminate under arterial pressure resulting in pockets of pooled blood and pressure build-up which results in the graft ballooning and bursting. This heterogeneity in the BC structure translates to varying mechanical properties along and across a single $\mathrm{BC}$ tube. Also, the bioreactors required for the production of the BC tubular structures are complex and difficult to operate if large scale is envisaged. Hence, a viable model for large scale BC graft production is yet to be developed.

$\mathrm{BC}$ is produced commercially in membranes or sheets with varying thicknesses and scale. We believe these methods for graft production, as presented to date, have an inherent inability to assure an economically viable and reproducible approach to the graft production. In this paper, we present a novel approach to small caliber BC graft $(<6 \mathrm{~mm})$ production that is both easily reproducible and provides a graft with adequate mechanical and biocompatible properties and, through in vivo experimentation, has already demonstrated promising patency.

\section{Experimental Section}

\subsection{Graft Fabrication}

\subsubsection{Bacterial Cellulose Production}

Bacterial cellulose was produced by Gluconacetobacter xylinus (ATCC 53582) in a modified Hestrin-Schramm medium, supplemented with $2 \%$ Corn Steep Liquor (Sigma-Aldrich, Germany) and $0.6 \%$ ethanol, at $\mathrm{pH}$ 5.0. The resulting $\mathrm{BC}$ membranes were then washed in $4 \%(\mathrm{w} / \mathrm{v}) \mathrm{NaOH}$. The membranes were then thoroughly washed with distilled water until $\mathrm{pH}$ was neutral.

\subsubsection{Endotoxin Removal Assay}

Endotoxin removal was performed as follows: quadruplicate BC samples $\left(1.5 \times 2.5 \times 4 \mathrm{~cm}^{3}\right)$ were placed in $200 \mathrm{ml}$ of distilled water (control) and 5\% SDS (w/v). The water and detergent solutions were changed after every $24 \mathrm{~h}$ over the course of $3 \mathrm{~d}$. Duplicate BC samples were taken daily after each washing step, washed abundantly with distilled water, frozen at $-80^{\circ} \mathrm{C}$ and freeze-dried, prior to endotoxin quantification. The samples were subsequently cut into samples of the same weight $20 \pm 4 \mathrm{mg}$ and rehydrated with $500 \mu$ l of apyrogenic water. The concentration of endotoxin in each sample was determined using the Pierce LAL Chromogenic Endotoxin Quantitation Kit (Thermo Scientific, Rockford, IL USA).

\subsection{Graft Manufacturing}

$\mathrm{BC}$ grafts were prepared by cutting large $\mathrm{BC}$ sheets $(1.5 \times 25 \times$ $30 \mathrm{~cm}^{3}$ ) into $1.5 \times 2.5 \times 7 \mathrm{~cm}^{3}$ blocks. These were then washed in 
$5 \%$ SDS (w/v) over $4 \mathrm{~d}$, with the detergent solution being exchanged every $24 \mathrm{~h}$, then washed abundantly with distilled water. The blocks were then perforated along the center of the sample with a sharp $4 \mathrm{~mm}$ (outer diameter) metallic needle and then dried by mass transfer of water from the hydrogel to blotting paper until a final wall thickness of roughly $2 \mathrm{~mm}$ was achieved. The resulting tubular structures were then freeze-dried and stored at room temperature until needed (see Figure 1). All grafts tested had a luminal caliber of $4 \mathrm{~mm}$ and independently of the final length used in the various assays were initially $7 \mathrm{~cm}$ long. The BC grafts used for in vivo experimentation were rehydrated in sterile $0.9 \%(\mathrm{w} / \mathrm{v})$ saline solution and autoclaved at $121^{\circ} \mathrm{C}$ for $20 \mathrm{~min} 72 \mathrm{~h}$ before surgery.

\subsection{Surface Profilometry}

Freeze-dried BC grafts were split open longitudinally and fixed with double-sided tape to glass slides. Porcine femoral artery, commercial ePTFE, and PET grafts were also split longitudinally and affixed the same way (PET grafts were stretched as much as possible in order to remove the kinking inherent to the graft). BC grafts, ePTFE, and PET were all tested dry while the porcine femoral artery was briefly paper-dried, to remove excess water, before analysis. No tensile strain was applied to the samples, other than that required to un-kink the PET grafts.

The sample surface profiles were then measured five times along the same surface (each measurement roughly $1 \mathrm{~mm}$ apart) with a KLC TENCOR D-100 profilometer along $20 \mathrm{~mm}$ (for porcine femoral artery, ePTFE, and PET) and $30 \mathrm{~mm}$ for BC at a set speed of $0.2 \mathrm{~mm} \cdot \mathrm{s}^{-1}$ and a resolution of 2000 points per $\mathrm{mm}$. The results were then plotted on a three-dimensional graph so to convey an idea of the three-dimensional surface of the each sample. Roughness was calculated as the amplitude between maximum peak height and minimum valley depth.

\subsection{Cryo-SEM Imaging}

Cross-sections of the BC grafts were imaged via Cryo-SEM on a JEOL JSM 6301F/Oxford INCA Energy 350/Gatan Alto 2500. The graft samples were prepared as described above and then cut into $1 \mathrm{~mm}$ wide sections and placed upright in a metallic support. The samples were then rapidly cooled by plunging them into sub-cooled nitrogen (slush nitrogen) and transferred under vacuum to the cold stage of the preparation chamber. The samples were then fractured, sublimated for $180 \mathrm{~s}$ at $-90^{\circ} \mathrm{C}$, and sputter coated with $\mathrm{Au} / \mathrm{Pd}$. The samples were finally transferred into the SEM chamber and imaged at $-150^{\circ} \mathrm{C}$. Pore size was estimated from the micrographs using the freeware ImageJ (National Institutes of Health, Maryland, USA). These measurements were performed using the mean diameter of a circle a total of 30 times over 4 micrographs.

\subsection{Mechanical Tests}

\subsubsection{Tensile Strength Determination}

BC grafts, along with unprocessed BC grafts (unBC grafts - not freeze-dried ones), ePTFE, and porcine femoral artery (PFA) were tested for their longitudinal tensile strength in quintuplicate $(n=5)$ on a Shimadzu AG-X $50 \mathrm{kN}$ at a traction speed of $5 \mathrm{~mm} \cdot \mathrm{min}^{-1}$ and a load cell of $1 \mathrm{kN}$. Each sample tested was split open longitudinally and the materials thickness $(2.43 \pm 0.24 \mathrm{~mm}, 2.36 \pm 0.19 \mathrm{~mm}$,
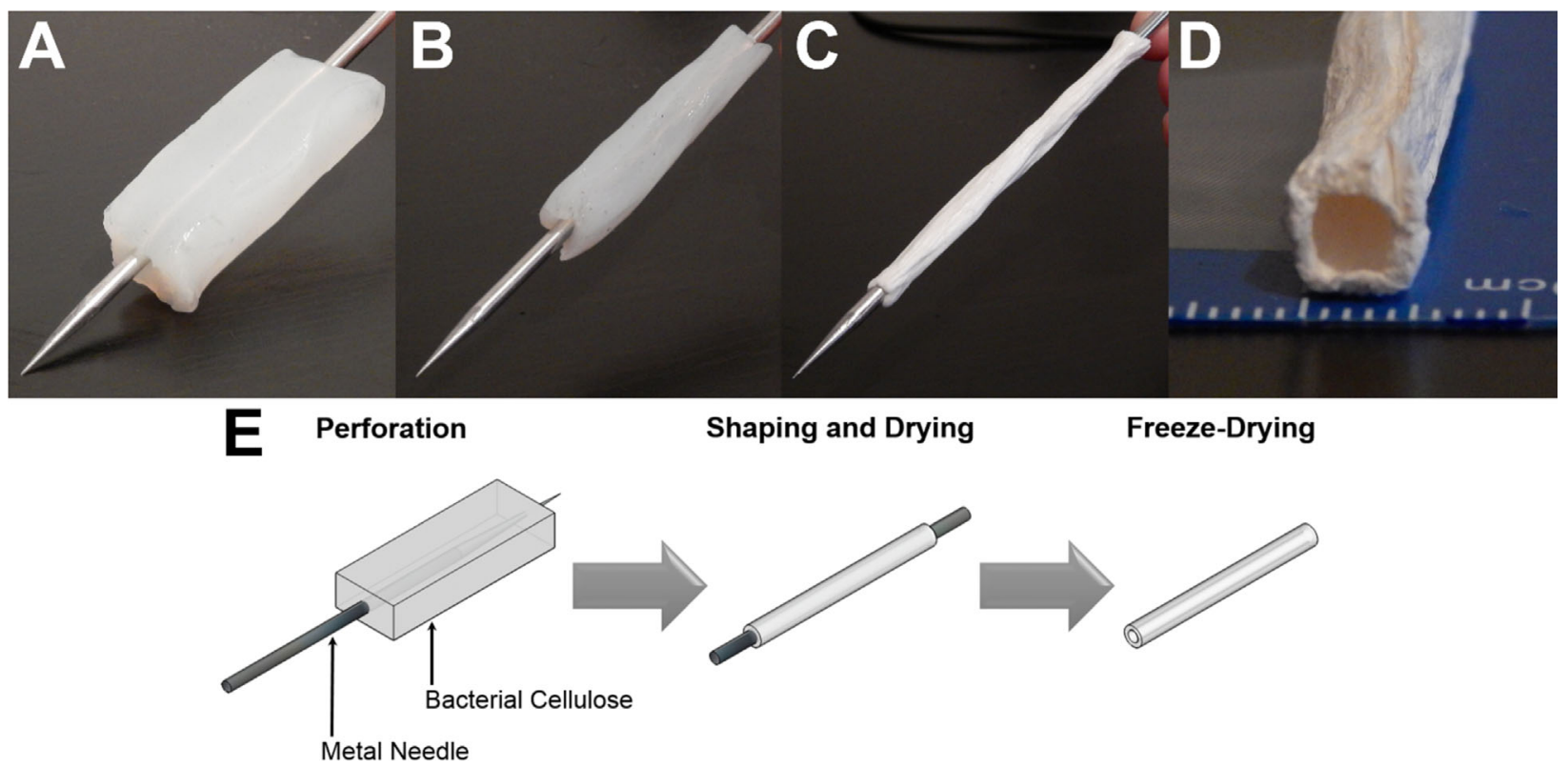

Figure 1. Photograph of the three stage process of graft production: (A) perforation, (B) shaping, (C) freeze-dried graft. (D) Top end view of a freeze-dried graft with $3 \mathrm{~mm}$ of luminal channel diameter. $(E)$ Schematic representation of the BC vascular graft formation process. From left to right: the BC perforated with a sharp metallic needle, dried by capillary action and shaped into a cylinder and finally freeze-dried.

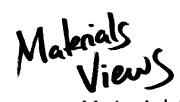

www.MaterialsViews.com 
$0.69 \pm 0.01 \mathrm{~mm}, 0.55 \pm 0.01 \mathrm{~mm}$ for $\mathrm{BC}$, UnBG, ePTFE, and PFA, respectively) and width $(14.67 \pm 0.57 \mathrm{~mm}, 17.90 \pm 0.55 \mathrm{~mm}$, $12.21 \pm 0.69 \mathrm{~mm}, 15.95 \pm 1.39 \mathrm{~mm}$ for $\mathrm{BC}$, UnBG, ePTFE, and PFA) was measured with digital calipers and accounted for in the calculations of the strength parameters. The distance between clamps was set to $25 \mathrm{~mm}$ for all samples tested.

$\mathrm{BC}$ and porcine femoral artery were tested wet and so special care was taken in order to prevent sample slipping from the hydraulic grips by using both fine grain sandpaper and construction paper. The same conditions to prevent slipping were used on the ePTFE samples. The Young's moduli were calculated automatically, using the equipment software, by linear fit over the linear deformation of each stress-strain curve and then averaged to obtain the material's Young's modulus.

\subsubsection{Suture Retention Assay}

Wet $\mathrm{BC}$ grafts with $6 \mathrm{~cm}$ length (4 mm internal diameter and $7 \mathrm{~mm}$ external diameter) were fixed the top clamp of a Z2.5 Zwick/Roell (Zwick GmbH \& Co. KG; Ulm; Germany). A 4-0 Prolene suture ( $0.15 \mathrm{~mm}$ diameter; Ethicon) was placed approximately $7 \mathrm{~mm}$ from the edge of the prosthesis and $2-3 \mathrm{~cm}$ from the top clamp (measured with a digital micrometer) and looped around the bottom clamp before clamping. The assay was then carried out in quintuplicate, on both freeze-dried and non-freeze-dried bacterial cellulose prosthetics, at a set speed of $10 \mathrm{~mm} \cdot \mathrm{min}^{-1}$ and a $1 \mathrm{~N}$ pre-load.

\subsubsection{Compliance Characterization}

Compliance characterization of $\mathrm{BC}$ grafts $(n=5)$ was performed using the system and method described previously by Diamantouros and colleagues. ${ }^{[34]}$ Briefly, the grafts were fixed into a chamber and initially exposed to a steady flow of purified water. A pulse $(1 \mathrm{~Hz})$ was added by a linear magnetic actuator. The pressures were stabilized at $80-120 \mathrm{mmHg}$. Variations in the outer diameter were measured by an optical micrometer sensor (LS-7030(M); Keyence Deutschland $\mathrm{GmbH}$, Neu-Isenburg, Germany) placed perpendicularly to the graft. Pressures were recorded by pressure transducers (CODAN pvd Critical Care GmbH; Forstinning, Germany). The measurements were realized for $30 \mathrm{~s}$ once the pressures were stable. Variations in the outer diameter and pressures were recorded by a LabVIEW application (National Instruments; Texas, USA). The circumferential compliance was then calculated with the following equation:

$$
C=\frac{\left(D_{\text {Syst }}-D_{\text {Diast }}\right) / D_{D_{\text {Diast }}}}{P_{\text {Syst }}-P_{\text {Diast }}} \times 100
$$

where $C$ is compliance $\left(\% \cdot \mathrm{mmHg}^{-1}\right), D$ is diameter $(\mu \mathrm{m})$, and $P$ is pressure (mmHg). "Syst" and "Diast" stand for the systolic and diastolic phase, respectively.

Additionally to the method presented by Kaźmierczak and Kazimierczak, ${ }^{[32]}$ ultrasound imaging was used (Vivid I, GE Healthcare; Freiburg, Germany) to monitor the changes of the internal diameter in response to pressure variations. Ultrasound videos were recorded from which still images were extracted and analyzed with ImageJ to determine the diameter of the lumen at three different positions along the longitudinal direction per image. Care was taken that the measurements from each video were performed at the same coordinates in each individual frame so as to account, as much as possible, for human error.

\subsection{In Vivo Experimentation and Histological Analysis}

\subsubsection{Surgical Procedure}

Three female domestic pigs (Sus scrofa domesticus) with weight ranging from $30-40 \mathrm{~kg}$ were restrained from food ( $24 \mathrm{~h}$ ) and water $(6 \mathrm{~h})$ before surgical procedure. All experiments were conducted according to the European Union Directive no.86/609/CEE for the use of animals in research and approved by the board for the ethical treatment of animals of the Faculty of Medicine of the University of Porto (ORBEA, FMUP, Oporto, Portugal). All procedures were performed under general anesthesia, with endotracheal intubation and spontaneous ventilation. A preanesthesia intramuscular injection of $32 \mathrm{mg} \cdot \mathrm{ml}^{-1}$ azaperone (Stressnil, Esteve Veterinaria) at a dose of $4 \mathrm{mg} \cdot \mathrm{kg}^{-1}$ reconstituted with $1 \mathrm{mg} \cdot \mathrm{ml}^{-1}$ midazolam (Dormicum, Roche) at a dose of $0.15-0.2 \mathrm{ml} \cdot \mathrm{kg}^{-1}$ was administered. Venous access was obtained through an intra-venous (IV) line placed in the marginal ear vein. Anesthesia was induced with $3 \mu \mathrm{g} \cdot \mathrm{kg}^{-1}$ fentanyl (Fentanest, Janssen-Cilag), and sodium thiopental $\left(12.5 \mathrm{mg} \cdot \mathrm{kg}^{-1}\right.$ IV initially and then to effect Tiopental 0.5 B Braun, B. Braun). After orotracheal intubation, the 7- to $7.5-\mathrm{mm}$ tube was connected to the anesthetic equipment (Ohmeda, Boc Health Care), and anesthesia was maintained with a mixture of isoflurane (1.5-2.5\%; Isoflo, Esteve Veterinaria) in 100\% oxygen and fentanyl at constant-rate infusion of $30-50 \mu \mathrm{g} \cdot \mathrm{kg}^{-1} \cdot \mathrm{h}^{-1}$. Afterward an epidural analgesia protocol was implemented with application of $1 \mathrm{mg} \cdot \mathrm{kg}^{-1}$ morphine (Morfina 1\% Braun, B. Braunl). To address infection prophylaxis, all animals received intramuscular injection of $1 \mathrm{~g}$ ceftriaxone (Rocephin, Roche) before surgery. End-tidal $\mathrm{CO}_{2}$, oxygen saturation, electrocardiography, and temperature were monitored continuously (Cygnus 1000C Vet Monitor, Servive Portugal). Pigs were placed in supine position and shaved, and electrocardiographic electrodes were attached to the chest for continuous evaluation of cardiac electrical function. Normal saline $\left(2-4 \mathrm{~mL} \cdot \mathrm{kg}^{-1}\right.$ hourly) was infused through the venous cannula in the auricular vein during surgery, to maintain adequate preload stability.

Open surgery was then performed with isolation of the common femoral artery, the profunda femoris artery and the superficial artery. All BC grafts used in this study were previously SDS washed, in order to remove endotoxin, as described above. The BC grafts, with $3-4 \mathrm{~cm}$ in length, were anastomosed in the left hind limb in a homolateral-femoral artery bypass. Papaverine was administered topically to the artery to avoid arterial spasm. The animals were heparinized systemically with an intravenous bolus injection of $200 \mathrm{U} \cdot \mathrm{kg}^{-1}$ of heparin (Heparina Sódica B. Braun, B.Braun), 5 min before clamping of the target arteries. BC grafts were then anastomosed with continuous suture with $6 / 0$ monofilament polypropylene sutures (Prolene, Ethicon). The femoral artery from the right hind limb was exposed in a sham-procedure. 
After the surgery, the pigs were transported back to their quarters, only when arterial oxygen tension stabilized above 95\%. Pigs were monitored until their body temperature normalized (with digital thermometers) and they were able to ambulate. They then were observed at least 2 or 3 times daily for locomotor activity, respiratory changes, body temperature, and food and water intake. Analgesia was maintained during the first $24 \mathrm{~h}$ after surgery by using meloxicam $\left(0.5 \mathrm{mg} \cdot \mathrm{kg}^{-1} \mathrm{IM}\right.$; Metacam, Boehringer Ingelheim). Acetylsalicylic acid (150 mg/ animal per os daily; Ácido Acetilsalicílico ratiopharm $100 \mathrm{mg}$, Ratiopharm) was given once daily until euthanasia, to prevent platelet aggregation.

After the designated times, the animals were sacrificed by IV KCl injection after sedation with azaperone reconstituted with midazolam administered IM prior to induction with sodium thiopental (12.5 mg $\cdot \mathrm{kg}^{-1}$ IV initially and then to effect) and the grafts and sham-operated arteries were harvested and histologically evaluated for signs of chronic inflammation, infection, foreign body responses, clot formation, cell ingrowth, and diameter via electron microscopy.

\subsubsection{Histology}

Tissues were preserved in formalin for a minimum of $4 \mathrm{~d}$ and were embedded in paraffin in an automated tissue processor (Microm STP 120). Tissues were then transferred to embedding workstation to obtain paraffin blocks. From these, sections $(2 \mu \mathrm{m})$ were cut on a rotary microtome (Leica RM 2035) and stained with Hematoxylin and Eosin (H\&E) for light microscopic examination. Observations and photographs were made using an Olympus BX51 microscope equipped with an Olympus camera.

\subsubsection{Immunohistochemistry}

Immunostaining of endothelial cells was performed using the primary antibody-Mouse Anti Pig CD31 (AbD Serotec; Oxford, England). The slides were immersed in $10 \mathrm{mM}$ sodium citrate ( $\mathrm{pH}$ 6.0) buffer, and heated in a water bath $\left(95-99^{\circ} \mathrm{C}\right)$ for antigen retrieval. Antigen visualization was done with the Novocastra Novolink Polymer Detection System (Leica Microsystems GmbH, Wetzlar, Germany) and involved the following steps: sections were incubated with $\mathrm{H}_{2} \mathrm{O}_{2}$ (3\%) for 10 min to eliminate endogenous peroxidase activity followed by a $5 \mathrm{~min}$ incubation with a protein blocking agent. Sections were subsequently incubated overnight at $4{ }^{\circ} \mathrm{C}$ with the primary antibody diluted at 1:100 with bovine serum albumin (BSA) (5\%), and on the following day, washed in TBSbuffered saline solution before incubation for $30 \mathrm{~min}$ with the secondary anti-mouse antibody system, using diaminobenzidine (DAB) as a chromogen, provided in the afore-mentioned kit.

\subsection{Statistical Data Analysis}

All data and statistical analysis was performed using both GraphPad Prism and Origin Pro 9 software. Statistical significances were determined via One-way and Two-Way ANOVA, in the case of the endotoxin removal assay, tests after verifying the Normal distribution $(p<0.05)$ of the data via the Kolmogorov-Smirnov test.

\section{Results}

\subsection{Graft Fabrication}

The novel methodology for the production of the BC graft is documented and represented schematically in Figure 1. The BC grafts were produced with needles that were purposely built, regularly sharpened, and exchanged in order to assure consistent perforation. Special care was taken during perforation in order to assure a smooth luminal surface. An unsharpened needle resulted in tears during the perforation with the appearance of "flaps" that could occlude or restrict flow through the graft. While all grafts presented in this work were $4 \mathrm{~mm}$ in diameter, other diameters can be easily achieved by using different needles.

The drying and shaping was performed with the needle in place with special care to avoid changes to the luminal diameter of the graft and an irregular topography of the external surface. The grafts were found to dry quickly during the freeze-drying process (a maximum of $2 \mathrm{~d}$ is required for complete drying) and the least irregular grafts were obtained the quicker this process was performed.

\subsection{Surface Profilometry}

Our results show that the surface roughness of BC is in the same range of that of porcine femoral artery (see Figure 2), ranging roughly $300 \mathrm{~nm}$ in both cases from highest peaks to lowest valley, with no statistically significant difference between them $(p>0.05)$. On the other hand, both ePTFE and PET show a much greater surface roughness with a distance of approximately 600 and $1600 \mathrm{~nm}$ between peaks and valleys, respectively.

\subsection{Cryo-SEM Imaging}

Several BC graft samples, and several different regions of each graft, were observed via Cryo-SEM so as to maintain the hydrated three-dimensional structure of the grafts; the images presented here are representative of those observations (Figure 3). The fibril distribution inside of our grafts showed markedly different structures throughout the graft wall that are well distributed throughout. In lower magnifications, dense sheets of BC fibers intersecting each other are visible and appear in wave-like patterns, parallel to the luminal surface of the graft (Figure 3A, B, and C). Dense sheets are surrounded by low density open porous regions (Figure 3D) with pore sizes ranging from 1 to $5 \mu \mathrm{m}$ in diameter. Additionally, there seems to be a consistent and dense single layer of $\mathrm{BC}$ fibers over the entire luminal surface of the graft that is immediately followed by open porous regions (Figure 3E).

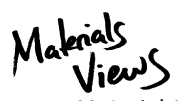

www.MaterialsViews.com 

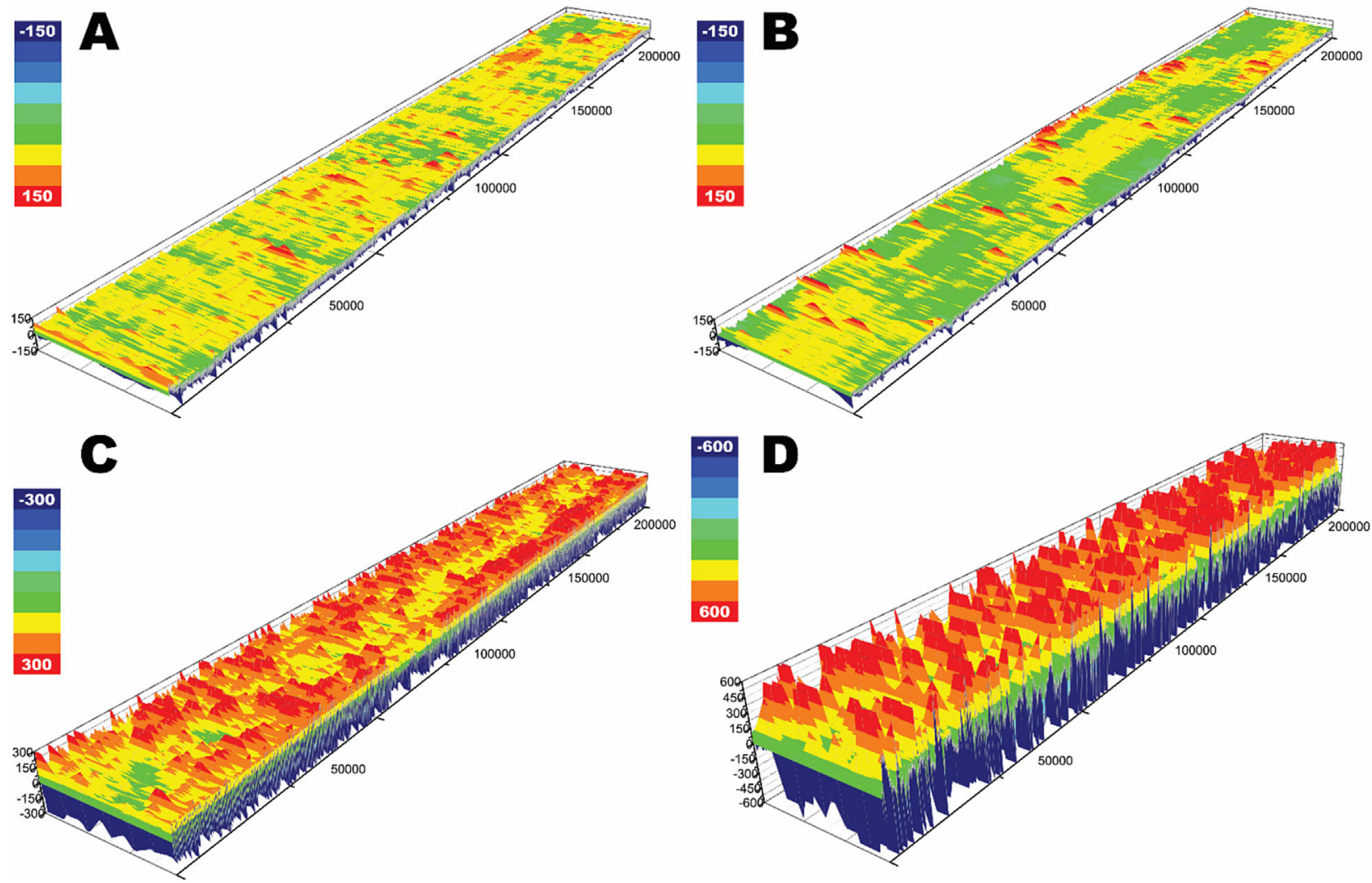

Figure 2. Surface profiles of Porcine Femoral Artery (A), BC graft (B), ePTFE graft (C) and a PET graft (D). The plots were obtained from five separate linear measurements (2000 points $\cdot \mathrm{mm}^{-1}$ ) over $2 \mathrm{~cm}(3 \mathrm{~cm}$ for $\mathrm{BC}$ ), spaced $1 \mathrm{~mm}$ apart and plotted in a three-dimensional graph.

\subsection{Endotoxin Removal Study}

Endotoxin (Lipopolysaccharide; LPS) concentration on water-washed and SDS-washed BC was determined with the Pierce LAL Chromogenic Endotoxin Quantitation Kit. Total LPS concentration on non-washed BC over the course of the study was $2.65 \pm 0.27 \mathrm{EU} \cdot \mathrm{ml}^{-1}$ (Endotoxin Units $/ \mathrm{ml}$ ) and dropped to $0.43 \pm 0.15,0.06 \pm 0.04$, and $0.003 \pm 0.02$ $\mathrm{EU} \cdot \mathrm{ml}^{-1}$ over the following $3 \mathrm{~d}$ (Figure 4). Total LPS concentration was shown to be significantly different between SDS-washed and water-washed BC $(p<0.05)$. Results also show a significant difference between a single and twice SDS-washed $(p<0.05)$ and no significant difference between the second and third washes.

\subsection{Mechanical Tests}

\subsubsection{Tensile Strength Determination}

Mechanical testing demonstrated marked differences between porcine femoral artery (PFA), ePTFE, BC and unBC grafts) - see Table 1 and 2 . The $B C$ and unBC grafts show no statistically significant difference between them $(p>0.05)$ in regards to any of the parameters. However, the BC graft did show higher values for maximum tensile strength
(Max Force), maximum stress and strain. PFA, while mechanically weak as compared to the other materials in regards to strength, was by far the most elastic, deforming $133 \%$ before rupture. Also noteworthy, ePTFE was shown to be most rigid of the tested materials, with the highest values in all the parameters. The BC grafts thus better resembles the biological vessels than ePTFE, in the sense that it presents higher elasticity, translated in a Young modulus in the same order of magnitude as PFA's.

\subsubsection{Suture Retention Strength and Compliance Characterization}

Results showed that both unBC and BC grafts demonstrated suture retention strength of $3.94 \pm 0.38$ and $4.20 \pm 0.99 \mathrm{~N}$, respectively, with no statistically significant difference between them $(p>0.05)$. Also, we determined BC graft compliance via laser detector and determined external compliance to be $0.4 \times 10^{-2} \% \cdot \mathrm{mmHg}^{-1}$.

\subsection{In Vivo Experimentation and Histological Analysis}

As a preliminary in vivo assay, 3 surgical procedures were performed to date, with implantation of the $B C$ graft in a 

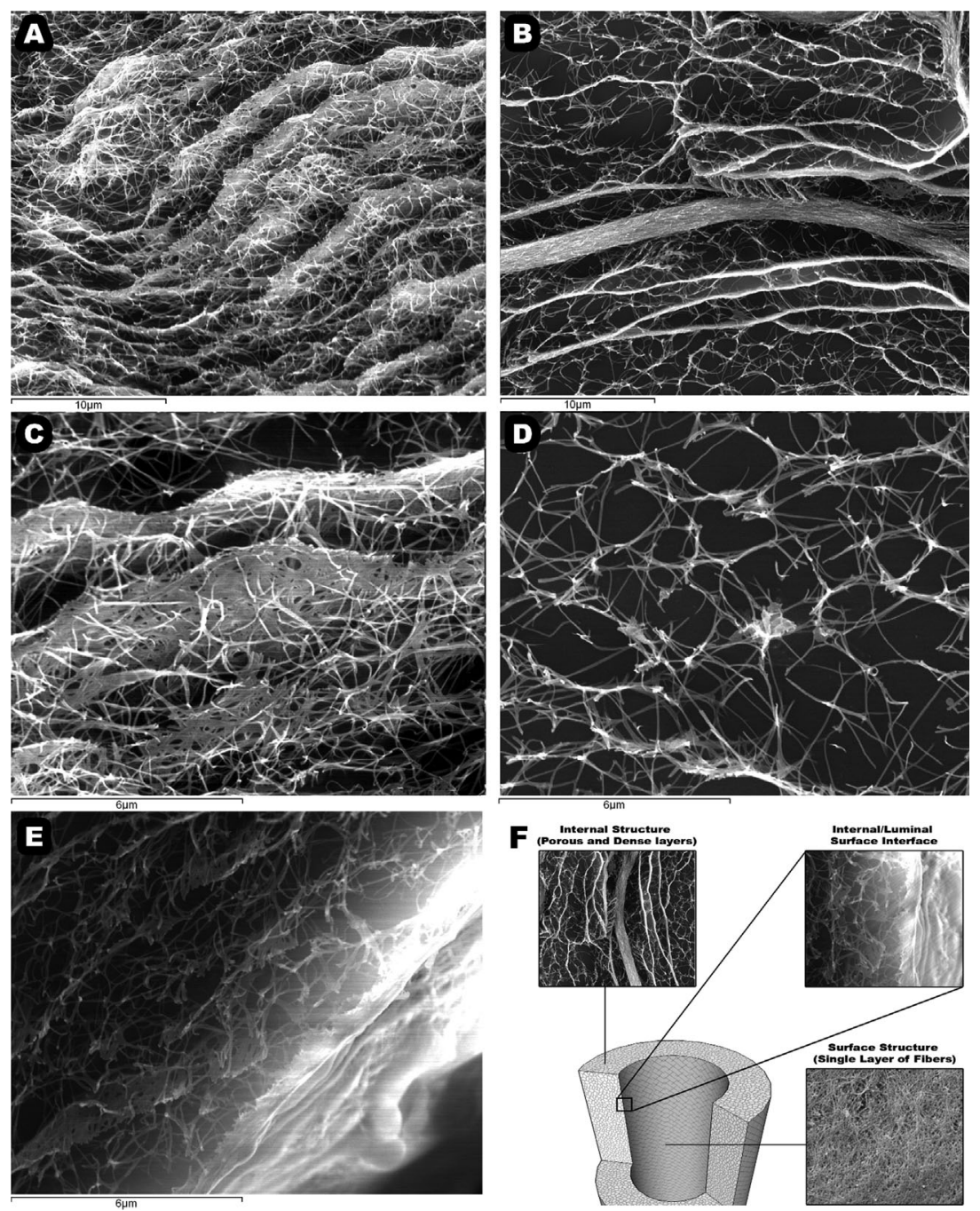

Figure 3. Cryo-SEM images of a portion of the cross-section of the graft wall. (A) and (B) $4000 \times$ magnification with noticeable dense regions layered between open-pore structures; (C) $10000 \times$ magnification of the dense BC regions; (D) $10000 \times$ magnification of the open-pore structures and (E) $10000 \times$ magnification of the edge of the luminal surface. homolateral-femoral artery bypass on the left hind limb of female domestic pigs (see Figure 5A). The right femoral artery was also isolated in a shamoperated procedure and served as a control for the implanted graft. No significant changes to the arterial wall of the control vessel were detected.

All three procedures presented confirmed patency after $1 \mathrm{~m}$. The first of these procedures was terminated one month post-op with blood flow confirmed via Doppler ultrasound prior to explantation, as also observed visually during tissue collection and later in the histological analysis. The final two procedures had a confirmed one month patency via Doppler ultrasound. However, these two grafts were found to be occluded two months post-op.

Histological analysis and immunohistochemistry staining were performed on the explanted grafts. All BC grafts were found intact and well integrated into the surrounding tissue with no significant fibrosis or macroscopic, external, evidence of inflammation. Histological analysis showed a distinct, three-layered, structure with cellular adhesion and infiltration on both the luminal and adventitial surfaces of the graft and an unpopulated and larger BC central region. All non-patent grafts were found to have an occlusive thrombus.

On the one-month patent graft, CD31 positive cells were detected on the luminal surface and are believed to be endothelial or progenitor endothelial cells that migrated from the adjoining femoral artery (Figure 5B and F). The presence of neo-vessel formation was detected in the cell population on both the adventitial and luminal surfaces (Figure $5 \mathrm{C}$ and $\mathrm{E}$ ). It should be noted that a significant unspecific background $\mathrm{BC}$ staining occurred, somewhat compromising the contrast of the images obtained. The specificity of the CD31 cells staining was confirmed in a cross-section of the native vessel and also it should be noted that the stained cells observed on the luminal surface of the grafts presented an endotheliallike morphology. Figure 5D is representative of the cellular infiltration and population found on the adventitial surface of all the recovered grafts. The cell populations in the adventitial regions were determined to be composed, mostly, of fibroblasts, macrophages, and some giant cells with a small population of lymphocytes also present.

Figure 4. Endotoxin (LPS) concentration in EU/ml (Endotoxin Units $\cdot \mathrm{ml}^{-1}$ ) as determined after washing the BC in $5 \%$ SDS and water (negative control).

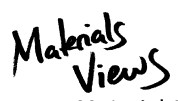

www.MaterialsViews.com 
Table 1. Mechanical test results for Porcine Femoral Artery (PFA), ePTFE, BC, and unBC grafts $(n=5)$.

\begin{tabular}{lcccc}
\hline Material & Young's modulus [N $\cdot \mathbf{~ m m}^{-\mathbf{2}}$ ] & Max force [N] & Max stress [N $\cdot \mathbf{~ m m}^{-\mathbf{2}}$ ] & Max strain [\%] \\
\hline PFA & $2.58 \pm 0.95$ & $10.05 \pm 3.39$ & $1.15 \pm 0.39^{*}$ & $133.46 \pm 11.25$ \\
ePTFE & $125.40 \pm 24.33$ & $198.54 \pm 13.33$ & $23.57 \pm 1.00$ & $40.62 \pm 9.92^{*}$ \\
BC graft & $10.56 \pm 1.53^{*}$ & $95.92 \pm 5.62^{*}$ & $2.75 \pm 0.38^{*}$ & $41.08 \pm 8.65^{*}$ \\
unBC graft & $10.70 \pm 2.23^{*}$ & $91.47 \pm 16.33^{*}$ & $2.10 \pm 0.26^{*}$ & $34.50 \pm 4.27^{*}$ \\
\hline
\end{tabular}

Values marked with an asterisk $(*)$ are not statistically different from each other in their respective columns $(p>0.05)$. Data are presented as mean \pm standard deviation.

Table 2. Suture Retention strength (N) of several autologous and synthetic grafts for tissue engineered blood vessels (TEBV), internal mammary artery (IMA), polytetrafluoroethylene (ePTFE), fibrin and fibrin/polylactic acid (PLA), human saphenous vein (HSV), collagen, and bacterial cellulose (BC).

\begin{tabular}{lccc}
\hline Graft & $\begin{array}{c}\text { Retention } \\
\text { strength [N] }\end{array}$ & SD & References \\
\hline IMA $^{\text {a) }}$ & 1.35 & \pm 0.49 & {$[49]$} \\
HSV $^{\text {a) }}$ & 2.60 & & {$[50]$} \\
TEBV $^{\text {a) }}$ & 1.49 & \pm 0.49 & {$[49]$} \\
Collagen $^{\text {b) }}$ & $0.82-2.87$ & $\pm 0.12-0.09$ & {$[51]$} \\
ePTFE $^{\text {c) }}$ & 4.70 & \pm 0.50 & {$[52]$} \\
BC $^{\text {d) }}$ & 4.20 & \pm 0.99 & - \\
unBC $^{\text {d) }}$ & 3.94 & \pm 0.38 & - \\
\hline
\end{tabular}

Suture retention strength for the $B C$ and $u n B C$ grafts are not statistically different $(p>0.05 ; n=4)$. The methodology used for suture retention measurements was as follows: ${ }^{\text {a) }} 2 \mathrm{~mm}$ bite at $2 \mathrm{~mm} \cdot \mathrm{min}^{-1},{ }^{\mathrm{b})} 2.5 \mathrm{~mm}$ bite at $2 \mathrm{~mm} \cdot \mathrm{s}^{-1},{ }^{\mathrm{c})} 3 \mathrm{~mm}$ bite at $20 \mathrm{~mm}$. $\min ^{-1},{ }^{d)} 7 \mathrm{~mm}$ bite at $10 \mathrm{~mm} \cdot \mathrm{min}^{-1}$; all performed with $5-0$ sutures.

\section{Discussion}

The method for BC graft production is hinged on its simplicity and a few steps, namely: perforation, simultaneous drying, and shaping and freeze-drying (Figure 1). Perforation of the graft by a sharp needle allows a precise control over the luminal diameter of the graft and directly affects the structure of the luminal surface of the graft. There is a consistent single dense layer of BC fibers over the entire luminal surface of the graft (Figure $3 \mathrm{E}$ ) that is immediately followed by an open porous regions. As the needle penetrates the cellulose block it pushes BC fibers out of its way and to the sides, the end result is a dense inner wall of BC fibers. This luminal surface plays an important role in containing the blood in the lumen of the graft while serving as a semi-permeable barrier to plasma and potentially adhered cells. Additionally, the surface created by this layer of BC fibers is quite smooth and, in regards to its profile, similar to that of a native artery (Figure 2). Nonuniform blood flow and shear stress have been shown to contribute to plaque formation. ${ }^{[35,36]}$ Physiological levels of shear maintain the homeostatic functioning of vessels and so, ideally, grafts should aim for physiological levels of shear. ${ }^{[37-41]}$ In this regard, the topography of both ePTFE and PET isquite different from that of both $\mathrm{BC}$ and the artery (Figure 2). The similarities between the surface roughness/ topography of the BC grafts and that of the arterial wall should theoretically match and allow for an un-interrupted and consistent blood flow over the transition from artery to graft maintaining physiological levels which, in the end, is beneficial to graft performance. It should be noted that care was taken to not apply any unnecessary tensile strain on the samples (other than that applied to PET grafts to un-kink the graft), to avoid artifacts.

The partial capillary drying, shaping, and freeze-drying steps are largely responsible for the final structural and mechanical properties of the resulting grafts. The drying and shaping allows the $\mathrm{BC}$ block to be shaped into a cylindrical tube with $2 \mathrm{~mm}$ in wall thickness. As a direct result, the graft wall density is brought from $0.02 \pm 0.01$ to $0.09 \pm 0.01 \mathrm{~g} \cdot \mathrm{cm}^{-3}$, with the final freeze-drying step being required to eliminate the remaining water. Theoretically, this allows individual BC fibers to come into contact with each other and form H-bonds, ${ }^{[42]}$ while maintaining each individual fiber's spatial orientation.

The contact and bonds created between fibers induced morphological changes in the BC hydrogel (Figure 3). BC has been extensively characterized as presenting an open porous structure that is relatively homogenous throughout the hydrogel. ${ }^{[16,42,43]}$ Our grafts present a dual structure, presenting dense sheets, formed by the collapse of fibers onto each other in the drying and with bonds formed during freeze-drying, (Figure 3A and B) layered in between the characteristic open porous morphology of BC (Figure 3D). These two distinct regions/structures complement each other structurally and functionally. The dense regions observed at a micro/sub-micro scale provide a slight 

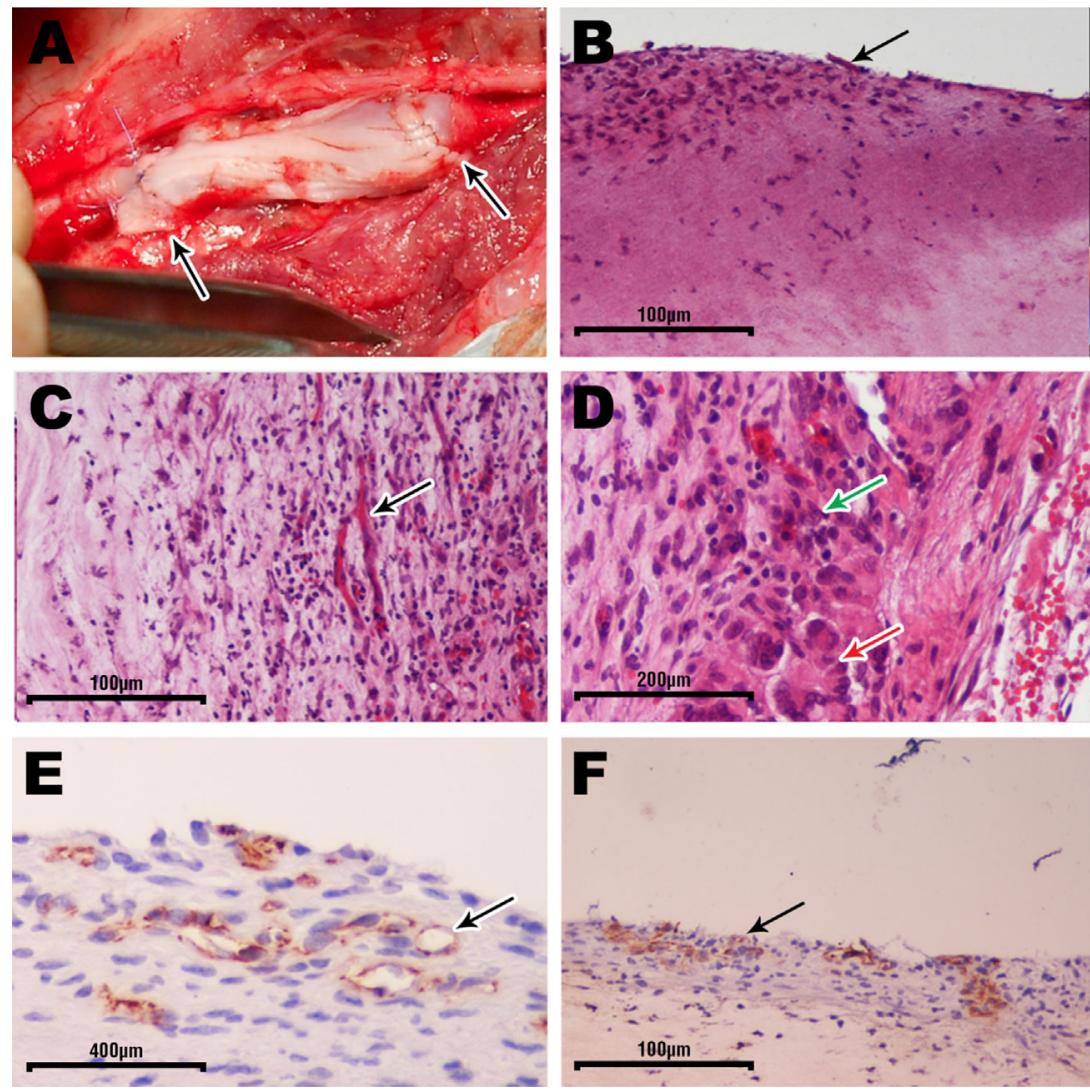

Figure 5. Representative photographs of the microscopy images of in vivo tests (A) Femoral-femoral artery BC graft placement, black arrows indicate anastomosis', (B) luminal surface of the graft with cell adhesion on the surface of the graft along and some cell infiltration, (C) external surface of BC graft showing formation of neo-vessels (arrow), (D) close-up detail of external surface of the graft with giant cells (red arrow) and macrophages (green arrow) among fibroblasts, (E) immunohistochemistry staining of $C_{31}$ positive cells forming neo-vessels on the luminal surface of the graft, $(F) C_{31}$ positive cell clusters on the luminal surface of the grafts.

increase in mechanical support, while allowing the graft to maintain the shape and structural integrity essential to a functional vascular graft. Simultaneously, the open-porous regions allow for void space and pore interconnectivity that in turn allows the graft to preserve its flexibility.

A final step in graft preparation was the removal of bacterial lipopolysaccharide (LPS or endotoxin) from the grafts by SDS wash. A significant amount of endotoxin $\left(2.65 \pm 0.27 \mathrm{EU} \cdot \mathrm{ml}^{-1}\right)$ remains on $\mathrm{BC}$ after alkaline wash, a standard procedure in purifying $B C$, which has been shown to induce a strong inflammatory response. ${ }^{[44]}$ The inflammatory response triggers coagulation mechanics and could subsequently cause thrombosis. A $24 \mathrm{~h}$ wash was enough to significantly reduce the overall concentration of LPS in BC by over $83 \%$ and a subsequent succession of two washes leaving only residual levels of LPS.

In terms of tensile strength, unBC and the BC grafts show no statistically significant difference between each other; though there seems to be a slight increase in the tensile strength of the $\mathrm{BC}$ grafts likely due to the higher number of bonds in the denser regions ( 95.92 vs. $91.47 \mathrm{~N}$ ). Since, the total number of BC fibers in a given crosssection is essentially identical the higher strength of the graft does not translate to significantly higher values. BC fibers are strong and inelastic ${ }^{[15]}$ as opposed to the native elastin which, has lower tensile strength and is capable of great deformation and shape memory. This is evident when comparing the tensile strength (95.92 vs. $10.05 \mathrm{~N}$ ) and elasticity, or deformation, (41 vs. 133\%) of the graft compared to a porcine vessel.

Similar to what happens with the tensile strength of the graft, suture retention of the $B C$ graft is influenced by the inelasticity of the $B C$ fibers when compared to that of native vessels. The large number of fibers, some organized in the stronger dense regions, provides a solid anchor point for the suture. This translates to a suture retention strength that is noticeably higher than that of native vessels, which as mentioned, tend to deform rather than resist the tensile stress. This suture retention strength is comparable to that of much stronger and rigid materials like ePTFE (see Table 2), though there are differences in the methodologies used that must be accounted for when comparing the results.

Another important consequence, of the distribution and layered structure of the graft wall is the compliance behavior of the graft. Intimal hyperplasia in both autologous saphenous vein and synthetic artery bypass grafts is a major reason for graft failure $\mathrm{e}^{[45]}$ and is closely associated with a mismatch in mechanical properties of the native vessel and the bypass graft. Diastolic and systolic pressure changes cause vessel walls to expand and contract. A mismatch in mechanical properties of native vessels and grafts adds direct stress to ingrown tissues. Specifically, this occurs at the point where the mechanical properties of the vessel wall changes. This can cause lesions to the cell layers which can expose underlying smooth muscle cells resulting in an abnormal migration and proliferation, along with the deposition of extracellular matrix, in the intimal layer (intimal hyperplasia). This leads to thickening of the vessel wall and causing a reduction in the lumen of the vessel, restricting blood flow which can eventually cause occlusion. ${ }^{[46]}$

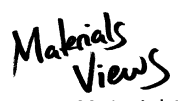

www.MaterialsViews.com 
Compliance was determined with a very precise method, using a laser detector and, results showed a value that would place the BC grafts in region of a non-compliant material $\left(0.4 \times 10^{-2} \% \cdot \mathrm{mmHg}^{-1}\right)$ (see Table 3$)$. This is an unexpected result suggesting that there would be a mismatch in mechanical properties between BC grafts and native arteries, somewhat contradictory with the experience one might have while manipulating the material, which appears to be rather biomimetic owing to the very high water content, fairly easy to expel by gentle pressure. Thus, our experience suggests that the malleability and flexibility of the graft, along with the wall structure observed in the SEM imaging, allows it to absorb the pressure change. We believe the graft wall retains a measure of compressibility and as such, a pulse would not be translated to the external surface of the graft. The open porous regions of the graft, as mentioned, provide void spaces that allow for fiber movement inside the graft wall in response to pressure changes.

The three implanted grafts were confirmed patent at one month post-op via Doppler ultrasound. One experiment was terminated at one month and the $\mathrm{BC}$ graft recovered in order to perform histological and immunohistochemical analysis. The other two procedures were terminated after two months when Doppler ultrasound confirmed no blood flow. These grafts were found occluded by thrombi. No evident macroscopic signs of inflammation were visible at the explantation. A large population of fibroblasts and macrophages, along with giant cells and a small population of lymphocytes could be observed in the thrombi. There appeared to be some blood flow, due to the presence of red

Table 3. Compliance values (SD) as found from the literature for internal mammary artery (IMA), human saphenous vein (HSV), human iliac artery (HIA), tissue engineered blood vessel (TEBV), polyurethane (PU), polyethylene terephthalate (PET), expanded polytetrafluoroethylene (ePTFE), bacterial cellulose/chitosan blend $(\mathrm{BC} / \mathrm{Chi})$ and measurements for the compliance of the $\mathrm{BC}$ grafts as measured externally $(n=5)$.

\begin{tabular}{|c|c|c|c|}
\hline Graft & $\begin{array}{c}\text { Compliance } \\
{\left[\times 10^{-2} \% \cdot \mathrm{mmHg}^{-1}\right]}\end{array}$ & SD & References \\
\hline IMA & 11.5 & \pm 3.9 & [49] \\
\hline HSV & $3.7-12.8$ & $\pm 1.3-1.9$ & [53] \\
\hline HIA & 8.0 & \pm 5.9 & [54] \\
\hline TEBV & $1.5-3.4$ & \pm 0.3 & {$[49,50]$} \\
\hline PU & 8.1 & \pm 0.4 & [54] \\
\hline PET & 1.8 & \pm 1.2 & [54] \\
\hline ePTFE & $1.2-1.6$ & \pm 0.3 & {$[45,54]$} \\
\hline $\mathrm{BC} / \mathrm{Chi}$ & 5.9 & \pm 2.7 & [45] \\
\hline BC graft & 0.4 & \pm 0.1 & - \\
\hline
\end{tabular}

blood cells and channels in the thrombi though flow would be severely restricted. No evident behavioral signs of occlusion could be observed in the live animals due to collateral circulation compensating for the restricted blood flow in the femoral artery. We believe that the occlusions we observed after two months of implantation are associated with handling of the animals to perform the ultrasound imaging after one month. The animals had to be handled in order to administer the sedative and due to their size and strength this process was somewhat rough and could have caused lesions to the anastomosis site. Further work is being conducted in order to ascertain if this is indeed true.

The results, in regards to in vivo experimentation, are still in their early stages yet, as compared to the findings of both Malm et al. ${ }^{[47]}$ and Wipperman et al. ${ }^{[48]}$ The luminal surface in this graft was largely populated by a cell population containing endothelial, or endothelial-like cells, a fibroblast population, along with some macrophages and giant cells, covered and infiltrated the adventitial surface. The occurrence of neo-vessel formation was found both, on the luminal and adventitial surfaces of the grafts. Cell infiltration and adhesion is largely limited to the surface and immediate vicinity of the graft, the interior of the BC graft wall remaining largely unpopulated. Both Malm et al. ${ }^{[4]}$ and Wipperman et al. ${ }^{[48]}$ reported similar findings though no neo-vessel formation was reported in their cases.

While coverage of the grafts adventitial surface was complete, the exact extension of surface coverage on the luminal surface could not be precisely determined. Also, the origin of the cells on the adventitial and luminal surfaces is undetermined. However, we believe these to be, in large part, cells that would have migrated from the anastomotic sites, at least in regards to the luminal surface, rather than circulating cells that adhered to the $\mathrm{BC}$ graft surface similarly to what was proposed by Malm et al. ${ }^{[47]}$

\section{Conclusion}

Several methods have been proposed and patented for BC graft production, all requiring dedicated bioreactors and production protocols. Our novel approach has allowed us to produce a dense, malleable, and mechanically strong tubular BC prosthesis. In vitro, the BC grafts herein presented seem to constitute a suitable alternative as synthetic vascular grafts, though in vivo experimentation has yet to confirm the viability of the $\mathrm{BC}$ grafts we here present.

This work is still ongoing mostly in order to expand upon the sample size of the in vivo tests, as well as, prolonging those same tests into longer implantation times in order to gain a more significant perspective of the feasibility of a $\mathrm{BC}$ grafts as a long-term, off-the-shelf, and cost-effective 
synthetic graft. Continued work will demonstrate any further limitations of the grafts, as is, and allow us to address possibilities such as nanocompositing the graft with other polymers, such as fibrin, or surface modifications, like the incorporation of heparin, in order to extend implantation times.

Acknowledgements: This study was supported by the Portuguese Foundation for Science and Technology (FCT) and the European Community fund FEDER, through Program COMPETE, under the scope of the Projects FCOMP-01-0124-FEDER-007025 (PTDC/AMB/ 68393/2006), PEST-OE/EOB/LA0023/2013, PEST-C/FIS/UI607/2013, RECI/BBB-EBI/0179/2012 (FCOMP-01-0124-FEDER-027462) and the Projects "BioEnv-Biotechnology and Bioengineering for a sustainable world" and "Matepro-Optimizing Materials and Processes". NORTE-07-0124-FEDER-000048, co-funded by the Programa Operacional Regional do Norte (ON.2-O Novo Norte), OREN, FEDER. The authors also acknowledge the fellowship awarded to Alexandre Felipe Leitão (SFRH/BD/66094/2009) funded by the Fundação para a Ciência e Técnologia (FCT). The authors also thank support by FCT through the project BCGrafts, FCOMP-01-0124-FEDER-014773 (PTDC/EBB/EBI/112170/2009) and by the People Program (Marie Curie Actions) of the European Union's Seventh Framework Program FP7/2007-2013/under REA grant agreement n317512.

Received: July 3, 2015; Revised: August 20, 2015; Published online: September 21, 2015; DOI: 10.1002/mabi.201500251

Keywords: bacterial cellulose; biocompatibility; endothelialization; mechanical properties; surface roughness; vascular grafts

[1] A. Leitão, I. Silva, M. Faria, M. Gama, in Encyclopedia of Biomedical Polymers and Polymeric Biomaterials, Taylor \& Francis, CRC Press, United Kingdom 2014.

[2] M. Wilhelmi, S. Jockenhoevel, P. Mela, Biomedizinische Tech. Biomed. Eng. 2014, 59, 185.

[3] J. Chlupac, E. Filova, L. Bacakova, Phys. Res. 2009, 58 Suppl 2, S119.

[4] M. Holmberg, X. Hou, Langmuir 2010, 26, 938.

[5] R. J. van Det, B. H. Vriens, J. van der Palen, R. H. Geelkerken, Eur. J. Vas. Endovas. Surg. 2009, 37, 457.

[6] G. J. Tsai, W. H. Su, J. Food Protect. 1999, 62, 239.

[7] S. Schopka, T. Schmid, C. Schmid, K. Lehle, Materials 2010, 3, 638.

[8] S. Ravi, E. L. Chaikof, Regener. Med. 2010, 5, 107.

[9] J. P. Stegemann, S. N. Kaszuba, S. L. Rowe, Tissue Eng. 2007, 13, 2601.

[10] J. G. Nemeno-Guanzon, S. Lee, J. R. Berg, Y. H. Jo, J. E. Yeo, B. M. Nam, Y.-G. Koh, J. I. Lee, J. Biomed. Biotechnol. 2012, 2012, 14.

[11] V. A. Kumar, J. M. Caves, C. A. Haller, E. Dai, L. Li, S. Grainger, E. L. Chaikof, Acta Biomater. 2013, 9, 8067.

[12] L. P. Amarnath, A. Srinivas, A. Ramamurthi, Biomaterials 2006, 27, 1416.

[13] S. Jockenhoevel, G. Zund, S. P. Hoerstrup, K. Chalabi, J. S. Sachweh, L. Demircan, B. J. Messmer, M. Turina, Eur. J. Cardiothorac. Surg. 2001, 19, 424.

[14] S. de Valence, J. C. Tille, D. Mugnai, W. Mrowczynski, R. Gurny, M. Moller, B. H. Walpoth, Biomaterials 2012, 33, 38.
[15] A. Leitão, J. Silva, F. Dourado, M. Gama, Materials 2013, 6, 1956.

[16] D. Klemm, D. Schumann, U. Udhardt, S. Marsch, Prog. Polym. Sci. 2001, 26, 1561.

[17] A. Svensson, E. Nicklasson, T. Harrah, B. Panilaitis, D. L. Kaplan, M. Brittberg, P. Gatenholm, Biomaterials 2005 , 26, 419.

[18] D. R. Solway, M. Consalter, D. J. Levinson, Wounds 2010, 22, 17.

[19] J. D. Fontana, A. M. de Souza, C. K. Fontana, I. L. Torriani, J. C. Moreschi, B. J. Gallotti, S. J. de Souza, G. P. Narcisco, J. A. Bichara, L. F. Farah, Appl. Biochem. Biotechnol. 1990, 24-25, 253.

[20] F. K. Andrade, R. Costa, L. Domingues, R. Soares, M. Gama, Acta Biomater. 2010, 6, 4034.

[21] A. F. Leitão, S. Gupta, J. P. Silva, I. Reviakine, M. Gama, Colloids and Surf. B Biointerfaces 2013, 111, 493.

[22] H. Bäckdahl, B. Risberg, P. Gatenholm, Mater. Sci. Eng. C 2011 , 31, 14.

[23] H. Backdahl, G. Helenius, A. Bodin, U. Nannmark, B. R. Johansson, B. Risberg, P. Gatenholm, Biomaterials 2006, 27, 2141.

[24] F. Andrade, R. Pertile, F. Douradoa, Cellulose Structure and Properties, Derivatives and Industrial Uses. Nova Science Publishers, Inc 2010, 427.

[25] A. Bodin, H. Bäckdahl, P. Gatenholm, L. Gustafsson, B. Risberg, Bacterial Cellulose Tubes, Google Patents, 2012.

[26] B. Dickerhoff, R. S. Farivar, M. L. Raghavan, V. Kumar, Vascular Prosthetic Assemblies, Google Patents, 2013.

[27] P. Gatenholm, H. Backdahl, T. J. Tzavaras, R. V. Davalos, M. B. Sano, Three-Dimensional Bioprinting of Biosynthetic Cellulose (bc) Implants and Scaffolds for Tissue Engineering, Google Patents, 2011.

[28] W. K. Wan, L. Millon, Poly(vinyl alcohol)-Bacterial Cellulose Nanocomposite, Google Patents, 2005.

[29] G. Serafica, C. Damien, F. Wright, H. Beam, Implantable Microbial Cellulose Materials for Various Medical Applications, Google Patents, 2007.

[30] H. Zahedmanesh, J. N. Mackle, A. Sellborn, K. Drotz, A. Bodin, P. Gatenholm, C. Lally, J. Biomed. Mater. Res. Part B 2011 97B, 105.

[31] K. Kowalska-Ludwicka, J. Cala, B. Grobelski, D. Sygut, D. Jesionek-Kupnicka, M. Kolodziejczyk, S. Bielecki, Z. Pasieka, Arch. Med. Sci. AMS 2013, 9, 527.

[32] D. Kaźmierczak, J. Kazimierczak, Fibres Text. East. Eur. 2010, $18,82$.

[33] A. Putra, A. Kakugo, H. Furukawa, J. P. Gong, Y. Osada, Polymer 2008, 49, 1885.

[34] S. Diamantouros, L. Hurtado-Aguilar, T. Schmitz-Rode, P. Mela, S. Jockenhoevel, Ann. Biomed. Eng. 2013, 41, 1979.

[35] K. S. Cunningham, A. I. Gotlieb, Lab. Invest. 2005, 85, 9.

[36] E. Cecchi, C. Giglioli, S. Valente, C. Lazzeri, G. F. Gensini, R. Abbate, L. Mannini, Atherosclerosis 2011, 214, 249.

[37] N. Resnick, H. Yahav, A. Shay-Salit, M. Shushy, S. Schubert, L. C. M. Zilberman, E. Wofovitz, Prog. Biophys. Mol. Biol. 2003, 81, 177.

[38] A. E. Silver, J. A. Vita, Circulation 2006, 113, 2787.

[39] V. Milleret, T. Hefti, H. Hall, V. Vogel, D. Eberli, Acta Biomater. 2012, 8, 4349.

[40] F. Shen, C. J. Kastrup, Y. Liu, R. F. Ismagilov, Arterioscler. Thromb. Vasc. Biol. 2008, 28, 2035.

[41] W. He, Z. Hu, A. Xu, R. Liu, H. Yin, J. Wang, S. Wang, Cell Biochem. Biophys. 2013, 66, 855.

[42] C. Clasen, B. Sultanova, T. Wilhelms, P. Heisig, W. M. Kulicke, Macromol. Symp. 2006, 244, 48.

[43] A. M. Sokolnicki, R. J. Fisher, T. P. Harrah, D. L. Kaplan, J. Membr. Sci. 2006, 272, 15.

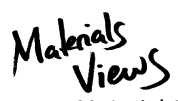

www.MaterialsViews.com 
[44] D. Heumann, T. Roger, Clin. Chim. Acta 2002, 323, 59.

[45] E. P. Azevedo, R. Retarekar, M. L. Raghavan, V. Kumar, J. Biomater. Sci. Polym. Ed. 2012, 24, 239.

[46] M. J. Thubrikar, Vascular Mechanics and Pathology, Springer, New York, United States 2007.

[47] C. J. Malm, B. Risberg, A. Bodin, H. Bäckdahl, B. R. Johansson, P. Gatenholm, A. Jeppsson, Scand. Cardiovasc. J. 2012, 46, 57.

[48] J. Wippermann, D. Schumann, D. Klemm, H. Kosmehl, S. Salehi-Gelani, T. Wahlers, Eur. J. Vasc. Endovasc. Surg. 2009, 37, 592.

[49] G. Konig, T. N. McAllister, N. Dusserre, S. A. Garrido, C. Iyican, A. Marini, A. Fiorillo, H. Avila, W. Wystrychowski, K. Zagalski, M. Maruszewski, A. L. Jones, L. Cierpka, L. M. de la Fuente, N. L'Heureux, Biomaterials 2009, 30, 1542.
[50] N. L'Heureux, N. Dusserre, G. Konig, B. Victor, P. Keire, T. N. Wight, N. A. Chronos, A. E. Kyles, C. R. Gregory, G. Hoyt, R. C. Robbins, T. N. McAllister, Nat. Med. 2006, 12, 361.

[51] S. Shah, Fabrication Of Small Diameter Vascular Graft Using Stacked Collagen Films, Masters Thesis, The University of Texas at Arlington 2013.

[52] M. Isaka, T. Nishibe, Y. Okuda, M. Saito, T. Seno, K. Yamashita, Y. Izumisawa, T. Kotani, K. Yasuda, Ann. Thorac. Cardiovasc. Surg. 2006, 12, 37.

[53] H. Zachrisson, M. Lindenberger, D. Hallman, M. Ekman, D. Neider, T. Länne, Clin. Physiol. Funct. Imaging 2011, 31, 300.

[54] N. R. Tai, H. J. Salacinski, A. Edwards, G. Hamilton, A. M. Seifalian, Br. J. Surg. 2000, 87, 1516. 